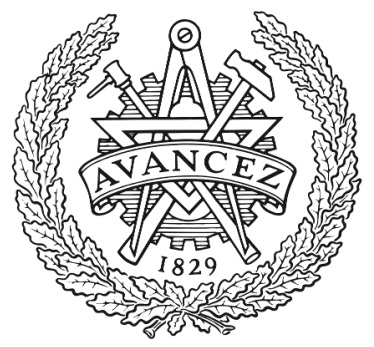

CHALMERS

UNIVERSITY OF TECHNOLOGY

\title{
First Principles Calculations of Palladium Nanoparticle XANES Spectra
}

Downloaded from: https://research.chalmers.se, 2023-04-26 08:42 UTC

Citation for the original published paper (version of record):

Nilsson, J., Carlsson, P., Grönbeck, H. et al (2017). First Principles Calculations of Palladium Nanoparticle XANES Spectra. Topics in Catalysis, 60(3-5): 283-288.

http://dx.doi.org/10.1007/s11244-016-0612-0

N.B. When citing this work, cite the original published paper. 


\title{
First principles calculation to interpret palladium XANES spectra
}

\author{
Johan Nilsson ${ }^{1,3 *}$, Per-Anders Carlsson ${ }^{1,3}$, Henrik Grönbeck ${ }^{2,3}$, and Magnus Skoglundh ${ }^{1,3}$ \\ ${ }^{1}$ Department of Chemistry and Chemical Engineering, Chalmers University of Technology, 41296 Göteborg, \\ Sweden \\ ${ }^{2}$ Department of Applied Physics, Chalmers University of Technology, 41296 Göteborg, Sweden \\ ${ }^{3}$ Competence Centre for Catalysis, Chalmers University of Technology, 41296 Göteborg, Sweden \\ *johan.nilsson@chalmers.se
}

\section{Abstract}

X-ray absorption spectroscopy is a common technique for in situ studies of catalysts. The interpretation of the near edge structure is, however, often hampered by lack of information of how structural and electronic contributions affect the spectra. Here, first principles calculations were used to explore effects of particle size, structural motif and oxidation state on the X-ray absorption near edge structure (XANES) for palladium nanoparticles (PdNP). In particular, the density functional theory in the implementation with planewaves and pseudo-potentials were used to structurally optimize PdNP with different size, structure and oxygen coverage. Pd K-edge XANES spectra were calculated using the realspace multiple scattering formalism. The results show that the Pd-Pd distances are slightly compressed for small NP leading to shifts in the XANES peak positions as compared to bulk $\mathrm{Pd}$. Moreover, the amplitude of the fine structure oscillations is found to increase with the average coordination number. The structural motif of the PdNP is found to influence the spectra in a minor fashion. Oxidation of the Pd surface increases the intensity in the XANES spectrum between the first and the second absorption peak, corresponding to the initial development of a whiteline peak. It is found that a strong whiteline peak only is developed for Pd-atoms with complete oxidation, which corresponds to coordination to four oxygen atoms.

\section{Introduction}

Supported palladium nanoparticles are used to catalyze a wide range of chemical reactions, where the oxidation of hydrocarbons and carbon monoxide in a three-way catalytic converter is but one example. For automotive applications, the active phase is often nanometer-sized palladium particles that are dispersed on a high surface area support material such as alumina. The size and morphology of the palladium nanoparticles (PdNP) are controlled by the synthesis method used to prepare the catalyst as well as by the interaction between palladium and the support material. Moreover, reaction conditions such as temperature and gas composition may affect the structure and composition of the active 
phase. Such changes has, for example, been observed during methane oxidation over $\mathrm{Pd} / \mathrm{Al}_{2} \mathrm{O}_{3}[1,2]$.

X-ray absorption fine structure (XAFS) has been established as an important technique for in situ characterization of heterogeneous catalysts as it enables monitoring of chemical and structural changes of the catalyst during the course of the catalytic reaction. The penetrating power of hard X-rays allows for use of in situ cells where the elements of interest for the catalytic reaction can be followed under gas exposure at temperatures that mimic realistic reaction conditions [3]. Furthermore, time-resolved measurements with sub-second time resolution can be performed using the quick XAFS or energy dispersive XAFS techniques. In the literature, in situ studies using XAFS have been reported for several reactions over palladium catalysts [1-8].

It has been shown that high-quality data of the extended fine structure (EXAFS) can allow for detailed analysis of the morphology of supported palladium nanoparticles [9]. However, the design of the in situ cell, the requirement of high time resolution, and the relatively high temperatures required to obtain appropriate kinetic data for the catalytic reaction, result in reduced spectroscopic quality compared to ex situ experiments performed at room temperature. The XANES region of the X-ray absorption spectra is typically more accessible for analysis, although this requires knowledge of how structural and electronic contributions affect the spectra

In this contribution we have investigated the XANES region of palladium K-edge X-ray absorption spectra using ab initio calculations. XANES spectra have been calculated for nanoparticle and single crystal surface model systems using the multiple scattering FEFF9 [10] code. The model systems were chosen to explore the effects of morphology, particle size, and oxidation state on the XANES region of Pd K-edge X-ray absorption spectra.

\section{Methods}

Three different types of model systems were considered, namely free nanoparticles, single crystal surfaces and bulk systems. The density functional theory (DFT) was applied with the gradient-corrected exchange-correlation functional according to Perdew, Burke, and Ernzerhof (PBE) [11]. In particular, the plane-wave pseudopotential code VASP was used [12-14]. The kinetic energy cutoff for the basis set was set to $460 \mathrm{eV}$ for systems with oxygen, whereas $320 \mathrm{eV}$ was used for bare Pd-systems. Standard PAW potentials [15,16] 
were used to treat the interaction between the valence electrons and the core. Reciprocal space integration over the Brillouin zone was approximated with finite sampling using Monkhorst-Pack grids $[17,18]$ corresponding to $12 \times 12 \times 12$ in the unit cell of bulk Pd and $10 \times 10 \times 8$ in the unit cell of bulk PdO. NP were treated in the $\Gamma$-point approximation. The Lattice parameters for bulk systems were determined by performing single point calculations at a set of different volumes whereas the geometries for the other systems were relaxed until the largest force in the system was smaller than $0.01 \mathrm{eV} / \AA \AA$.

Palladium K-edge XANES spectra were calculated using the FEFF9 multiple scattering code [10]. The electronic structure was in this case solved self-consistently within a radius around the absorbing atom using the local density approximation with the Hedin-Lundquist approximation [19]. The absorption spectra were calculated considering full multiple scattering (FMS) within a specified radius. Converged results were obtained with at least 55 atoms within the SCF radius and at least 135 atoms within the FMS radius. For nanoparticles with less than 55 atoms, all positions were within the SCF and FMS radii. For palladium nanoparticles spectra were calculated with the absorbing atom at each symmetry nonequivalent position and then a weighted average was taken over the different positions. For bulk systems and surfaces only a single absorbing atom was considered.

\section{Results and Discussion}

Five different structural motifs were used for the PdNP, namely octahedra $(19,44$, 85 , and 146 atoms), truncated octahedra $(38,79,116$, and 140 atoms), cuboctahedra (13, 55, and 147 atoms), ino-decahedra (55 atoms) and icosahedra (55 atoms). The considered motifs are shown in Figure 1. The octahedral NPs have eight (111) facets. Truncation of the vertices leads to the truncated forms which also have (100) facets. Further truncation results in the cuboctahedral motif which is characterized by having triangular (111) facets. The inodecahedral and icosahedral motifs have five-fold symmetry where the ino-decahedron has both (111) and (100) facets whereas the icosahedron only has (111) facets. 


\section{Structural motifs}
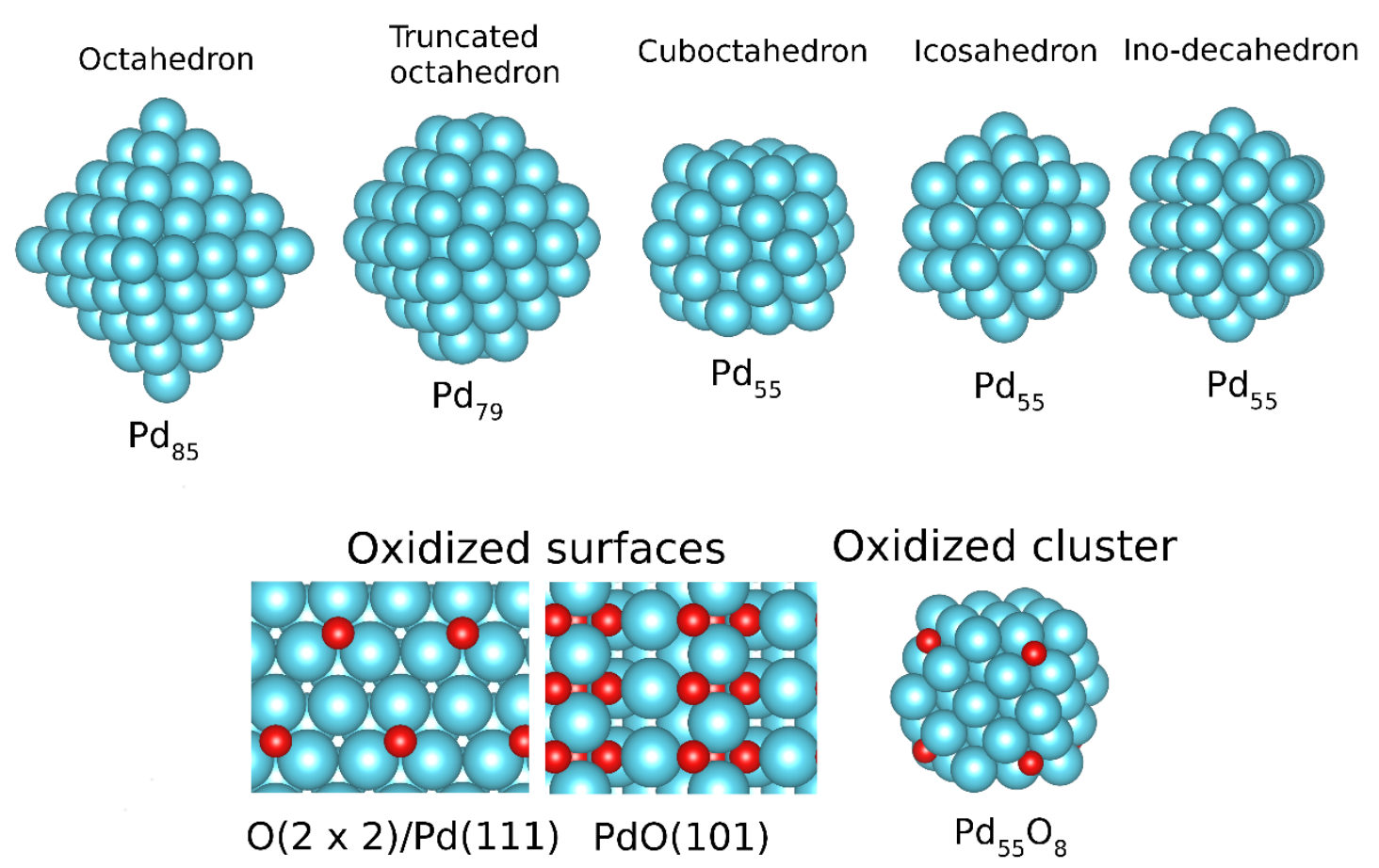

Figure 1: Structural motifs of bare PdNP. Also shown are the oxygen covered $O(2 \times 2) / P d(111)$ surface, the $\mathrm{PdO}(101)$ surface, and the oxygen covered $\mathrm{Pd}_{55} \mathrm{O}_{8}$ cuboctahedral PdNP.

Considering first the electronic and structural properties of bare NP, we focus on the octahedral forms of the PdNP. The electronic density of states for the $\mathrm{Pd}_{13}$ cuboctahedron and bulk Pd are shown in Figure $2 a$ and $2 b$, respectively. The $\mathrm{Pd}_{13}$ cuboctahedron is highly symmetric and the degeneracy of the electronic states is high. The electronic structure has molecular character with discrete states. In particular, the cluster has a clear HOMO-LUMO separation of $0.7 \mathrm{eV}$. For bulk Pd, the d-band is cut by the Fermi-energy. The $\mathrm{d}$-band for the cluster is not as wide as for the bulk. Another difference between $\mathrm{Pd}_{13}$ and Pd-bulk is the location of the sp-states which are stabilized in the case of the bulk system.

Turning to the structure of the octahedral forms of PdNP, both the average coordination number and the average Pd-Pd bond distance increase with increasing particle size which is shown in Figure 1c. Considering all Pd-Pd bond distances in the cluster the correlation is approximately linear $\left(r(P d-P d)(\AA)=2.627+0.014 N_{a v}, R^{2}=0.970\right)$ and is extrapolated to $2.795 \AA$ for the bulk coordination number $N=12$. The lattice constant in the bulk was calculated to be $3.955 \AA$, which corresponds to a Pd-Pd bond distance of $2.797 \AA$ (experimental lattice constant $3.89 \AA$ [20]). Considering the Pd-Pd bond distance just between the outer shell atoms at the surface, there is considerable spread between the 
different particles. The cohesive energy of the PdNP increases with increasing particle size as shown in Figure $1 \mathrm{~d}$. The fitted correlation is $\mathrm{E}_{\mathrm{coh}} / \mathrm{n}(\mathrm{eV})=3.83-3.63 \mathrm{n}^{-1 / 3}\left(R^{2}=0.988\right)$, which is extrapolated to a value of $3.83 \mathrm{eV}$ for bulk $\mathrm{Pd}$. The calculated cohesive energy for the bulk is $3.71 \mathrm{eV}$ whereas the experimental value is $3.91 \mathrm{eV}$ [21]. Similar sets of PdNP of different sizes has been investigated by Krüger at al. [22] and Yudanov et al. [23] and similar linear correlations between the average bond distance and cohesive energy have been found.
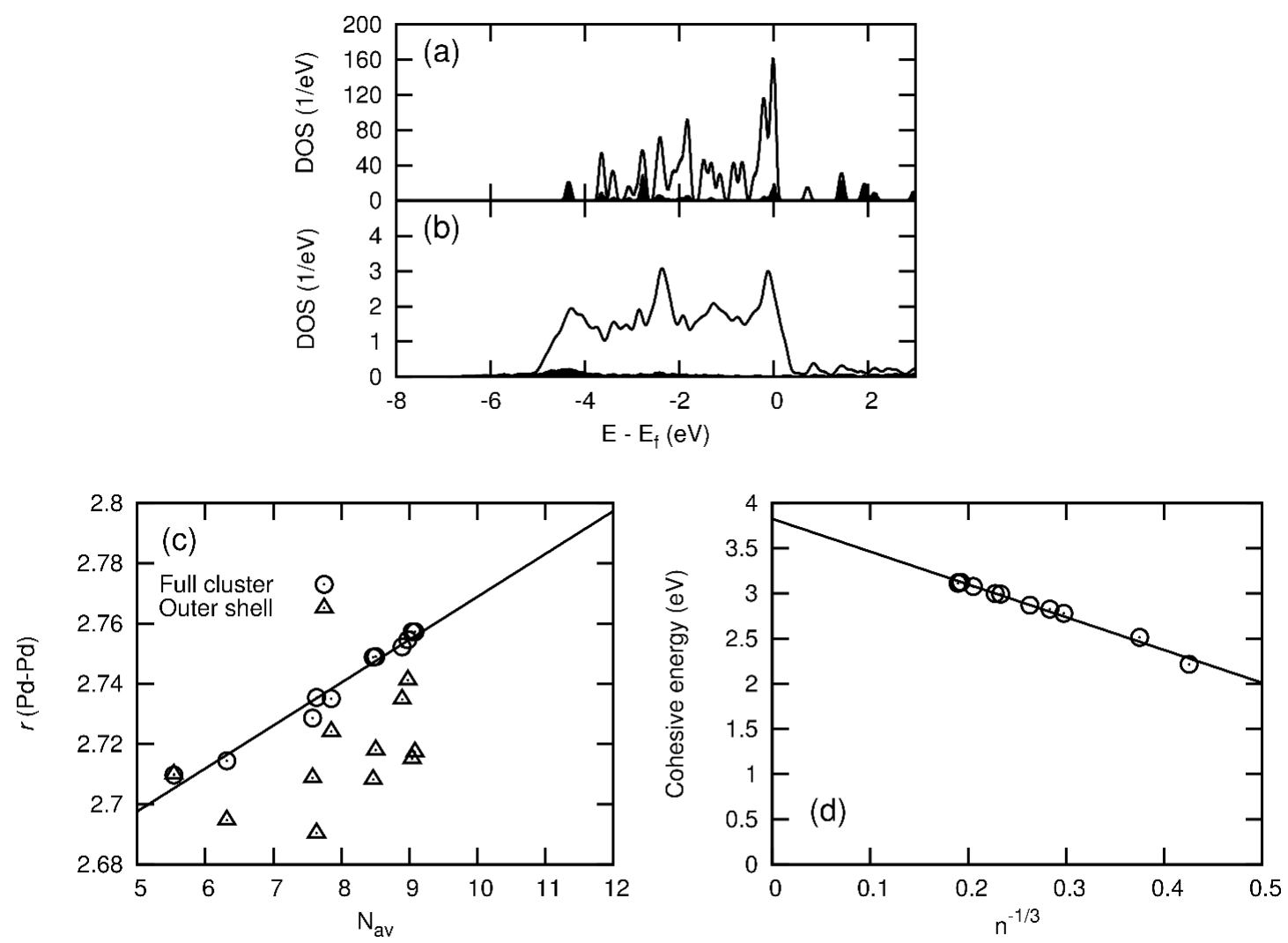

Figure 2: Electronic density of states for (a) $\mathrm{Pd}_{13}$ cuboctahedral nanoparticle, and (b) bulk Pd. The shaded area is the projection on the $s$ and p-states. (c) Average Pd-Pd distance $r(P d-P d)$ as a function of average coordination number $\mathrm{Nav}_{\mathrm{v}}$ for all cluster atoms (circles) and only outer shell (surface) atoms (triangles). (d) Cohesive energy $E_{c o h} / n$ of $P_{n}$ clusters as a function of $n^{-1 / 3}$.

Pd K-edge X-ray absorption spectra were calculated for the cuboctahedral NP with 13, 55 and 147 atoms to investigate the effect of particle size. Moreover, spectra were also calculated for the 55 atom icosahedron and ino-decahedron to allow comparison of different structural motifs. To investigate the effects of oxidation, the $\mathrm{Pd}(111)$ surface with an oxygen coverage of $0.25 \mathrm{ML}$ together with the $\mathrm{PdO}(101)$ surface were used. In addition to the results from oxidized surfaces, oxygen atoms were adsorbed on the (111) facets of the $\mathrm{Pd}_{55}$ cuboctahedron. Similar systems have previously been used as model systems for carbon monoxide adsorption on extended surfaces [23]. These structures are shown in Figure 1. 
Figure 3 shows the Pd K-edge XANES spectra calculated for the cuboctahedral palladium clusters containing 13,55 , and 147 palladium atoms. The calculated XANES spectra of palladium nanoparticles are similar to that of bulk $\mathrm{Pd}$ already for these small cluster sizes. The first two absorption maxima after the absorption edge are assigned to $5 p$ and $4 f$ type final states, respectively [24]. The $\mathrm{Pd}_{13}$ cuboctahedron has a spectrum which is similar to that of the larger nanoparticles although the absorption maxima are shifted to higher energies, the $4 f$ peak is shifted with $1.7 \mathrm{eV}$ compared to $\mathrm{Pd}_{147}$. The $\mathrm{Pd}_{55}$ nanoparticle has an equivalent peak shift of $0.8 \mathrm{eV}$. Contracted bond distances have been observed experimentally using EXAFS for large Pd particles with diameters of 7, 12 and $23 \mathrm{~nm}$, respectively [25]. A similar trend has been observed for palladium carbide [26] and palladium hydride [5], where peaks in the XANES region were shifted to lower energies due to an expansion of the lattice compared to the bulk.

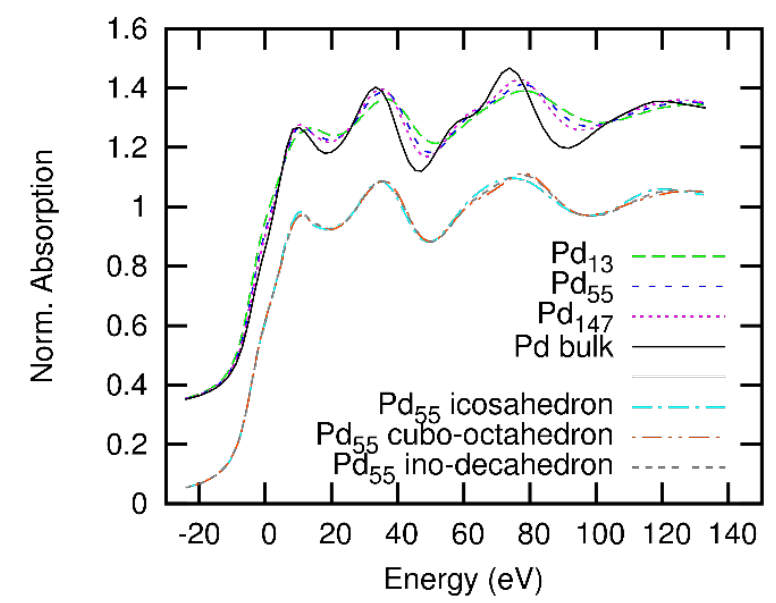

Figure 3: Calculated Pd K-edge XANES spectra of bulk Pd and cuboctahedral palladium clusters with 13, 55 , and 147 atoms (upper spectra), and cuboctahedron, icosahedron, and ino-decahedron with 55 palladium atoms (lower spectra).

To investigate the effect of particle morphology, XANES spectra were calculated for palladium nanoparticles with three different structural motifs: the cuboctahedron, the icosahedron, and the ino-decahedron, all containing 55 atoms. These spectra are shown in Figure 3. All three spectra show high resemblance indicating that the packing of the atoms has a limited impact on the shape of the XANES spectra. It should be noted that the essential features in the XANES spectra are similar for all $4 \mathrm{~d}$ transition metals, also the metals with a different packing than fcc such as molybdenum (bcc) and zirconium (hcp) [24], which is in line with the findings for the different nanoparticle morphologies in the present study.

Elucidating the structure of oxidized palladium nanoparticles is challenging for particles in the size range of tens of atoms as global structural optimization is prohibitively expensive with first-principles DFT calculations. To study oxidation, we decided to focus on single 
crystal surfaces where oxidized structures have been studied extensively in the past, both theoretically and experimentally.

On Pd(111), oxygen preferentially adsorbs in fcc hollow sites and forms a $(2 \times 2)$ ordered overlayer with a surface coverage of $0.25 \mathrm{ML}$ [27]. Higher oxidation leads eventually to complete oxidation into $\mathrm{PdO}$. We have considered bulk PdO together with $\mathrm{PdO}(101)$ facet, see Figure 1. XANES spectra were calculated for one of the palladium atoms forming the hollow site with oxygen adsorbed and also for the corresponding position on a bare $\operatorname{Pd}(111)$ surface, these spectra are shown in Figure 4. Adding the oxygen to the hollow results in a reduction in intensity of the $4 f$ peak and an increase in intensity of the $5 p$ peak, and the area between these two peaks.

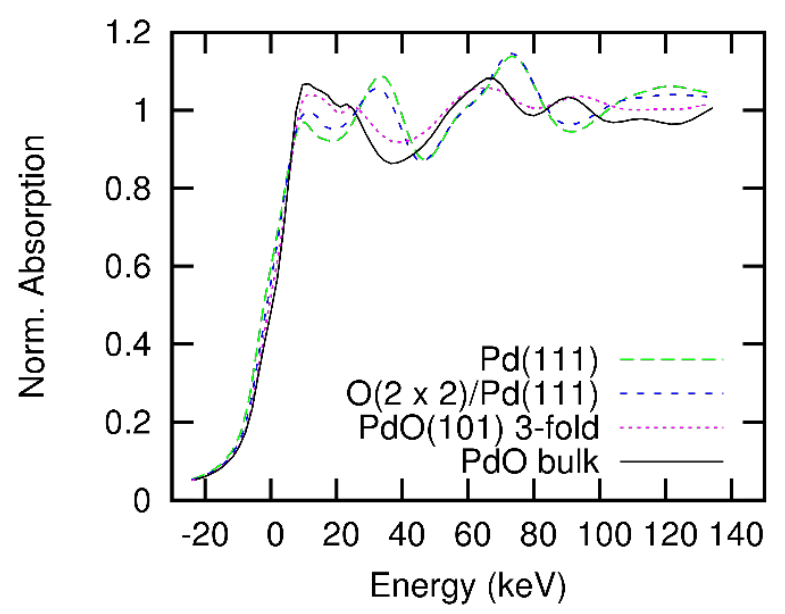

Figure 4: Pd K-edge XANES spectra for a palladium atom forming an fcc hollow in the $\mathrm{Pd}(111)$ surface, the corresponding atom with a $(2 \times 2)$ oxygen overlayer (coverage $0.25 \mathrm{ML}$ ), the 3-fold coordinated surface $\mathrm{Pd}$ in $\mathrm{PdO}(101)$, and bulk PdO.

Larger palladium nanoparticles adopt a polyhedral shape according to the Wulff construction. The oxidation of such nanoparticles is associated with an increase in area of the (100) surface as it becomes more stable upon oxygen adsorption [28,29]. The oxidation of the $\mathrm{Pd}(100)$ surface starts with the formation of a surface oxide consisting of a single $\mathrm{PdO}(101)$ monolayer. Continued oxidation results in epitaxial growth of several layers of $\mathrm{Pd}(101)$ forming a bulk like structure. The $\mathrm{PdO}(101)$ surface has palladium atoms that coordinate to four oxygen atoms like atoms in the bulk and also undercoordinated Pd atoms coordinating to three oxygen atoms. The calculated XANES spectrum for the undercoordinated $\mathrm{Pd}$ atoms in the $\mathrm{PdO}(101)$ surface is shown in Figure 4 and also the calculated XANES spectrum for palladium in bulk palladium oxide. The addition of oxygen neighbors to palladium successively results in a whiteline shape similar to that of bulk palladium oxide. 
To calculate a spectrum which is more representative of an experimental XANES spectrum, a cuboctahedral 55 atom palladium cluster with one oxygen atom adsorbed at the center of each (111) facet was used. The spectrum was calculated including the contribution from all non-equivalent palladium sites. This spectrum is shown in Figure 5 together with the spectrum for the corresponding bare Pd cluster. Qualitatively the spectrum is similar to the spectrum calculated for oxygen adsorbed on the $\mathrm{Pd}(111)$ surface.

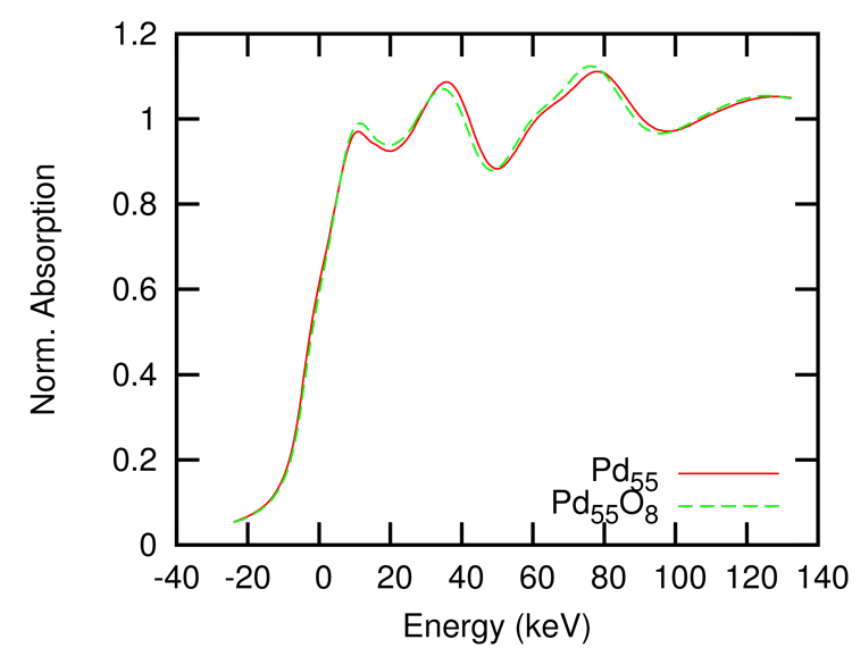

Figure 5: Calculated Pd K-edge XANES spectra for the cuboctahedral $\mathrm{Pd}_{55}$ cluster and the same cluster after absorption of 8 oxygen atoms at the center of the (111) facets.

\section{Conclusions}

In summary, ab initio XANES spectra have been calculated for a variety of palladium model systems relevant to catalysis. Nanoparticles of different sizes display a shift in the second peak after the absorption edge with a shift to lower energies as the size of the cluster decreases. This is due to the contraction of the Pd-Pd bond lengths in the small palladium clusters. For palladium clusters with different structural motifs the changes in the calculated XANES spectra are limited. Addition of oxygen in the coordination shell of palladium atoms causes a progressive change in the XANES spectra approaching that of bulk palladium oxide. The changes in the XANES spectra just from adsorption of oxygen on the surface of palladium nanoparticles is fairly limited, a bulk like local structure is necessary to have a strong whiteline peak.

One of the major strengths of $a b$ initio XANES spectra is the possibility to calculate spectra specific to particular particle configurations. In a supported catalyst different domains in the catalyst can be oxidized to different extents and since XAFS is a bulk sensitive technique, the recorded spectrum will represent an average of all local environments of the absorbing atoms. Calculating XANES spectra from first principles provides a method to easily access the XANES spectrum of a particular configuration. 


\section{Acknowledgements}

This work has been performed within the project "Unravelling catalytically active sites with X-ray absorption spectroscopy", Swedish Research Council (621- 2011-5009); the Röntgen-Ångström Collaboration "Catalysis on the atomic scale", Swedish Research Council (349-2011-6491); and partly within the Competence Centre for Catalysis, which is hosted by Chalmers University of Technology and financially supported by the Swedish Energy Agency and the member companies AB Volvo, ECAPS AB, Haldor Topsøe A/S, Scania CV AB, Volvo Car Corporation AB, and Wärtsilä Finland Oy.

\section{References}

[1] J. Nilsson, P.-A. Carlsson, S. Fouladvand, N.M. Martin, J. Gustafson, M.A. Newton, E. Lundgren, H. Grönbeck, M. Skoglundh, ACS Catal. (2015) 2481-2489.

[2] S.K. Matam, M.H. Aguirre, A. Weidenkaff, D. Ferri, J. Phys. Chem. C 114 (2010) 9439-9443.

[3] J.-D. Grunwaldt, M. Caravati, S. Hannemann, a. Baiker, Phys. Chem. Chem. Phys. 6 (2004) 3037.

[4] S. Reimann, J. Stötzel, R. Frahm, W. Kleist, J.-D. Grunwaldt, A. Baiker, J. Am. Chem. Soc. 133 (2011) 3921-3930.

[5] M. Bauer, R. Schoch, L. Shao, B. Zhang, A. Knop-Gericke, M. Willinger, R. Schlögl, D. Teschner, J. Phys. Chem. C 116 (2012) 22375-22385.

[6] D. Ferri, M.A. Newton, M. Nachtegaal, Top. Catal. 54 (2011) 1070-1078.

[7] K. Föttinger, J. a. Van Bokhoven, M. Nachtegaal, G. Rupprechter, J. Phys. Chem. Lett. 2 (2011) $428-$ 433.

[8] H. Feng, J.W. Elam, J. a. Libera, W. Setthapun, P.C. Stair, Chem. Mater. 22 (2010) 3133-3142.

[9] V. V. Srabionyan, A.L. Bugaev, V. V. Pryadchenko, L. a. Avakyan, J. a. Van Bokhoven, L. a. Bugaev, J. Phys. Chem. Solids 75 (2014) 470-476.

[10] J.J. Rehr, J.J. Kas, F.D. Vila, M.P. Prange, K. Jorissen, Phys. Chem. Chem. Phys. 12 (2010) 55035513.

[11] J.P. Perdew, K. Burke, M. Ernzerhof, Phys. Rev. Lett. 77 (1996) 3865-3868.

[12] G. Kresse, J. Hafner, Phys. Rev. B 49 (1994) 14251-14269.

[13] G. Kresse, J. Furthmüller, Comput. Mater. Sci. 6 (1996) 15-50.

[14] G. Kresse, J. Furthmüller, Phys. Rev. B 54 (1996) 11169-11186.

[15] P.E. Blöchl, Phys. Rev. B 50 (1994) 17953-17979.

[16] G. Kresse, D. Joubert, Phys. Rev. B 59 (1999) 1758-1775.

[17] J.D. Pack, H.J. Monkhorst, Phys. Rev. B 16 (1977) 1748-1749.

[18] H.J. Monkhorst, J.D. Pack, Phys. Rev. B 13 (1976) 5188-5192.

[19] L. Hedin, B.I. Lundqvist, J. Phys. C Solid State Phys. 4 (1971) 2064-2083.

[20] C. Nordling, J. Österman, Physics Handbook for Science and Engineering, 8th ed., Studentlitteratur, Lund, 2006.

[21] D.D. Wagman, W.H. Evans, V.B. Parker, R.H. Schumm, I. Halow, S.M. Bailey, K.L. Churney, R.L. Nuttall, J. Phys. Chem. Ref. Data Suppl. 11 (1982) 1-407.

[22] S. Krüger, S. Vent, F. Nörtemann, M. Staufer, N. Rösch, J. Chem. Phys. 115 (2001) 2082-2087.

[23] I. V. Yudanov, R. Sahnoun, K.M. Neyman, N. Rösch, J. Chem. Phys. 117 (2002) 9887.

[24] J.E. Muller, O. Jepsen, O.K. Andersen, J.W. Wilkins, Phys. Rev. Lett. 40 (1978) 720-722.

[25] C.-M. Lin, T.-L. Hung, Y.-H. Huang, K.-T. Wu, M.-T. Tang, C.-H. Lee, C. Chen, Y. Chen, Phys. Rev. B 75 (2007) 1-6.

[26] J.A. McCaulley, Phys. Rev. B 47 (1993) 4873-4879.

[27] E. Lundgren, G. Kresse, C. Klein, M. Borg, J.N. Andersen, M. De Santis, Y. Gauthier, C. Konvicka, M. Schmid, P. Varga, Phys. Rev. Lett. 88 (2002) 246103.

[28] C. Popa, T. Zhu, I. Tranca, P. Kaghazchi, T. Jacob, E.J.M. Hensen, Phys. Chem. Chem. Phys. 17 (2015) 2268-2273.

[29] H. Graoui, S. Giorgio, C.R. Henry, Surf. Sci. 417 (1998) 350-360. 